\title{
Annotated catalogue of the Haliplidae of China with the description of a new species and new records from China (Coleoptera,Adephaga)
}

\author{
Fenglong Jia ${ }^{1, \dagger}$, Bernhard van Vondel ${ }^{2,+}$ \\ I Institute of Entomology, Life Science School, Sun Yat-sen University, Guangdong, China 2 Natural History \\ Museum Rotterdam. plo: Roestuin 78, 3343 CV Hendrik-Ido-Ambacht, the Netherlands \\ † urn:lsid:zoobank.org:author:AD2EFC37-9653-4965-894A-97317A616A48 \\ † urn:lsid:zoobank.org:author:22362F62-5BA3-4D17-B528-013BEF43101E \\ Corresponding author: Fenglong Jia (fenglongjia@yahoo.com.cn)
}

Academic editor: Martin Fikácek | Received 1 June 2011 | Accepted 13 September 2011 | Published 5 October 2011

urn:lsid:zoobank.org:pub:9D9C8B9F-C541-42BF-8439-4AE9864F2469

Citation: Jia F, van Vondel B (2011) Annotated catalogue of the Haliplidae of China with the description of a new species and new records from China (Coleoptera, Adephaga). ZooKeys 133: 1-17. doi: 10.3897/zookeys.133.1642

\begin{abstract}
A revised checklist of Haliplidae (Coleoptera: Adephaga) of China is presented. A new species Haliplus (Haliplus) latreillei sp. n. is described from Guizhou, China. Three species, Haliplus (Haliplidius) confinis Stephens, Haliplus (Haliplus) ruficollis (De Geer) and Haliplus (Haliplus) sibricus Motschulsky are reported from China for the first time. Haliplus dalmatinus Müller is excluded from the list of Chinese species. A number of new provincial records from China is presented.
\end{abstract}

\section{Keywords}

Coleoptera, Haliplidae, Haliplus, new species, new records, China

\section{Introduction}

Haliplidae is a very small family of Coleoptera. A total of five genera and 238 species (Vondel 2005, 2007, 2009, 2010, Vondel and Spangler 2008, Watts and McRae 2010) have been described in the world of which only Haliplus Latreille, 1802 (with subgenera Liaphlus Guignot, 1928, Haliplidius Guignot, 1928 and Haliplus s. str.) and

Copyright Fenglong Jia, Bernhard van Vondel. This is an open access article distributed under the terms of the Creative Commons Attribution License, which permits unrestricted use, distribution, and reproduction in any medium, provided the original author and source are credited. 
Peltodytes Régimbart, 1879 (subgenus Peltodytes s.str.) are known in China. Wu (1937) reported two genera and 13 species from China, of which only 10 species are valid now. Since the description of Haliplus diruptus Balfour-Browne, 1947 from Tianjin, no new species were described from China until Vondel (1990) described Haliplus harminae Vondel, 1990 from Hubei. Subsequently Vondel (1991, 1992, 1995, 1998, 2003a) described five new species from China and revised all known Chinese species. Makhan (1999) described further three new species from China, Peltodytes aschnae Makhan, 1999, Haliplus rishwani Makhan, 1999 and Haliplus amrishi Makhan, 1999, which were all treated as synonyms of Peltodytes sinensis (Hope, 1845), Haliplus japonicus Sharp, 1873 and Haliplus sharpi Wehncke, 1880 respectively by Vondel (2003b). Štastný and Boukal (2003) described Haliplus rejseki Štastný \& Boukal, 2003 from Sichuan, being the first representative of the subgenus Haliplidius in China. Jia (2003) reported Haliplus (Liaphlus) dalmatinus Müller, 1900 (which identification is changed to $H$. abbreviatus Wehncke, 1880 in this paper) and Jia (2003) and Vondel (2003b) reported Peltodytes (Peltodytes) caesus (Duftschmid, 1805) from China.

The studies of the material of the Haliplidae deposited in the Coleoptera collection of the Sun Yat-sen University in Guangzhou, China and in several European collections result in a considerable extension on the knowledge of Chinese Haliplidae. This justifies a review of the species known so far from China, specified to the provinces in which they are found. In the present paper one new species of Haliplus is described and other three species of Haliplus are reported as new for China. Including this study 29 species are now known from China.

\section{Material and methods}

The present paper is based predominantly on 600 specimens of the family Haliplidae deposited in the Coleoptera collection of the Sun Yat-sen University (SYSU) in Guangzhou (Guangdong, China). All these specimens were identified by the first author and 25 specimens were re-examined by the second author. The second author examined another 210 specimens from the National Museum Prague, Czech Republic (NMPC); Naturhistorisches Museum Basel, Switzerland (NHMB), the Snow Entomological Collections of the University of Kansas, Lawrence, Kansas, USA (SEMC), the collection of F. Angelini, Brindisi, Italy (CA), the collection of H. Fery, Berlin, Germany $(\mathrm{CF})$, the collection of A. Nilsson, Umeå, Sweden $(\mathrm{CN})$ and second author's own collection $(\mathrm{CV})$.

Details on distribution are partly adopted from Vondel $(2005,2007)$. The genera Haliplus and Peltodytes were redescribed in detail by Holmen (1987). The key to genera was given by Vondel (1995). Morphological terminology largely follows Holmen (1987), Vondel (1991, 1992, 1993) and Vondel et al. (2006). Photographs were taken using a Zeiss Axioskop 40 compound microscope and a Leica M205C stereomicroscope combined with AutoMontage software. 


\section{Systematics}

Haliplus (Haliplus) latreillei sp. n.

urn:Isid:zoobank.org:act:1C3B87DA-E87E-40CA-B74D-2E537338AFEB

http://species-id.net/wiki/Haliplus_(Haliplus)_latreillei

Figs $1-6$

Type material. Holotype $\widehat{\partial}$, China, Guizhou, Guiyang, 6.x.1940, lgt. Zhe-long Pu (translation, labeled in Chinese) (SYSU). Paratypes (2 exs.): 1 o , same data as holotype (SYSU); 1 , same data as holotype (NMW).

Description. Length $2.9-3.0 \mathrm{~mm}$, width $1.6-1.7 \mathrm{~mm}$. Body oval, tapering backwards, widest before the middle (Fig. 1).

Head. Dark brown, somewhat lighter between eyes, anterior margin of clypeus densely punctured, but with much stronger and sparser punctures between eyes. Labrum yellowish brown with dark spot in the middle. Distance between eyes $1.6 \times$ width of one eye. Antennae light yellowish brown, not darkened apically. Palpi yellowish brown.

Pronotum. Yellow to yellowish brown. Without basal plicae, strongly and densely punctured. Lateral sides margined, straight to slightly convex. Base a little narrower than elytra at base.

Elytra. Yellowish brown, with dark interrupted lines on primary punctures rows, darkened along suture, with vague dark marks connecting primary puncture rows, without dark band basally. Completely margined. Primary puncture rows moderately strong and dense, 38-39 punctures in row 1 . Secondary punctures moderately strong and dense along suture, moderately strong and much sparse on intervals. All punctures darkened.

Ventral side. Brown red, with legs and anterior 1/fifth of prosternal process yellow brown; elytral epipleura yellowish brown with strong darkened punctures, reaching to abdominal sternite 6. Prosternal process narrowed between coxae, grooved along each side, anterior edge not margined, with moderately strong punctation. Metaventral process slightly bulbous with a row of strong punctures on each side that is slightly impressed, else moderately punctured (Fig. 2). Metacoxal plates reaching to fifth sternite, moderately strongly punctured, near suture weakly punctured, row of setae on posterior edge (Fig. 3). Fifth and sixth abdominal sternite each with sparse transverse puncture row. Last abdominal sternite weakly punctured in apical portion. No setiferous striole present on dorsal face of hind tibia, longer tibial spur of hind legs with dense teeth on inner side, about $2 / 3 \times$ length of first metatarsomere.

Males. Pro- and mesotarsomeres moderately widened and provided with suctionpads. Mesotarsomere 1 not very strongly excised. Penis and parameres as Figs 4. 5. 6.

Female. Unknown.

Etymology. The species is named in honour of Pierre André Latreille (1762-1833), a French entomologist who firstly used Haliplus as the genus name in 1802. 


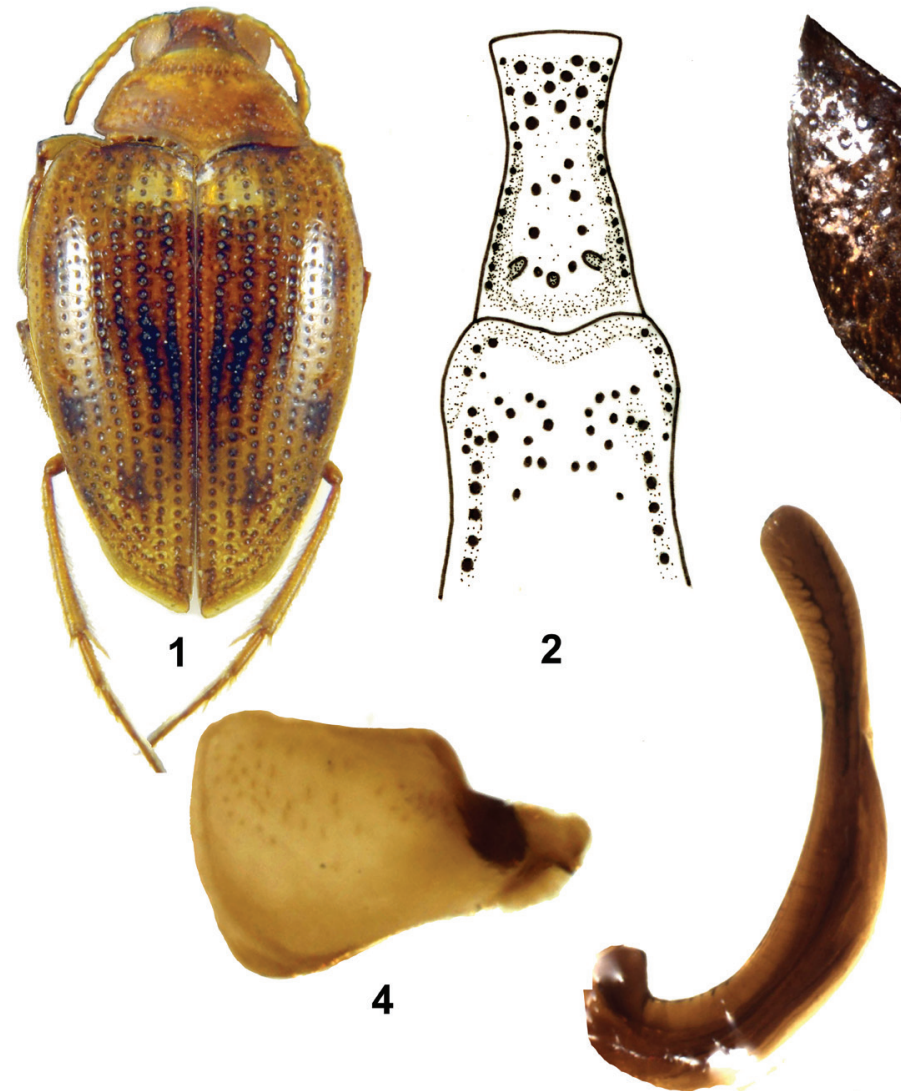

5
6

Figuress I-6. Haliplus latreillei sp. n. I habitus $\mathbf{2}$ prosternal and metaventral process $\mathbf{3}$ metacoxal plate 4 left paramere 5 penis $\mathbf{6}$ right paramere.

Differential diagnosis. This species is close to $H$. japonicus Sharp, 1873 and $H$. regimbarti Zaitzev, 1908 in body size and shape, arrangement of elytral dark spots and black lines, punctuation and the row of setae on posterior edge of the metacoxal plates. However, the new species lacks pronotal basal plicae, its pronotum lacks the transverse basal rim and it differs from the above species in the shape of the median lobe and parameres of the aedeagus. Despite the absence of pronotal plicae this species clearly belongs to the subgenus Haliplus s. str. due to the absence of the metatibial setiferous striole.

Distribution. Only known from the type locality. 


\section{List of Chinese Haliplidae}

Only valid names are given in this list. For complete synonymy and distribution outside China see Vondel (2005)

1. Haliplus (Haliplidius) confinis Stephens, 1828

http://species-id.net/wiki/Haliplus_(Haliplidius)_confinis

Figs $7-10$

Material examined. Xinjiang: $1 \widehat{\partial}$, Kashi, 1.viii.2006. lgt. Ling Zhao.

Distribution. Widely distributed western and north-eastern Palaearctic species reaching the utmost north-west of China: Xinjiang. New to China.

2. Haliplus (Haliplidus) rejseki Štastný et Boukal, 2003

http://species-id.net/wiki/Haliplus_(Haliplidus)_rejseki

Distribution. Endemic to China: Sichuan.

3. Haliplus (Haliplus) aliae Vondel, 2003

http://species-id.net/wiki/Haliplus_(Haliplus)_aliae

Distribution. Endemic to China: Tianjin.

4. Haliplus (Haliplus) furcatus Seidlitz, 1887

http://species-id.net/wiki/Haliplus_(Haliplus)_furcatus

Material examined. Heilongjiang: 2 exs., , Mishan, 26.viii.1964, lgt. De-ai Deng \& Shou-fa Hou. Inner Mongolia: 12 exs., Hailar, 23-26.vii.2003, lgt. Feng-long Jia (2 exs. CV); 1 ex., Huhenuor, 21-25.vii.2005, lgt. Feng-long Jia.

Distribution. Western and north-eastern Palaearctic species reaching China in the north-east: Heilongjiang, Inner Mongolia. New record for Inner Mongolia. 


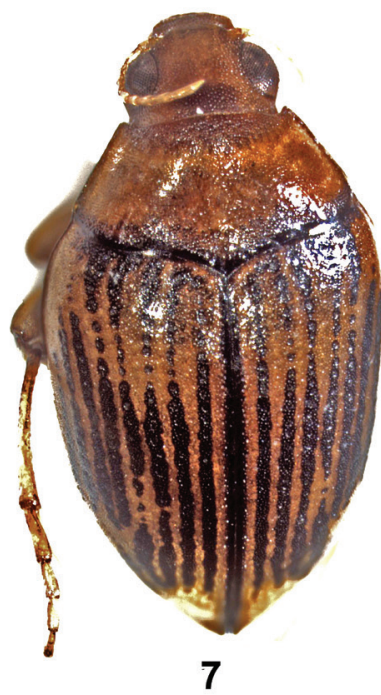

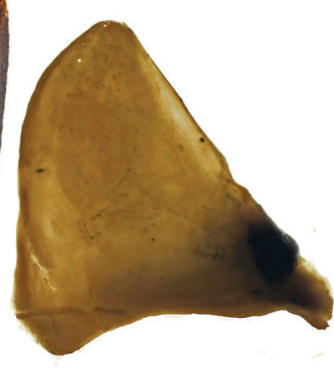

8

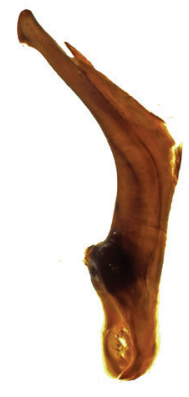

9

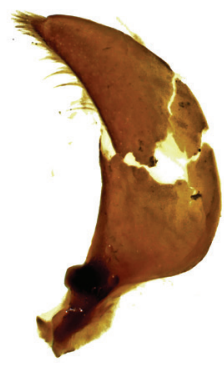

10

Figuress 7-10. Haliplus confinis $\mathbf{7}$ habitus $\mathbf{8}$ left paramere $\mathbf{9}$ penis $\mathbf{1 0}$ right paramere.

\section{Haliplus (Haliplus) harminae Vondel, 1990}

http://species-id.net/wiki/Haliplus_(Haliplus)_harminae

Material examined. Hunan: 1 q., Nanyue Mt., 2.ix.1941, lgt. Zhe-long Pu; Shaanxi: 1 o., Chang'an, Weiqu, 12.viii.1984, lgt. Zhi-he Huang.

Distribution. Endemic to China: Hubei, Hunan, Shaanxi. New records for Hunan and Shaanxi.

\section{Haliplus (Haliplus) japonicus Sharp, 1873}

http://species-id.net/wiki/Haliplus_(Haliplus)_japonicus

Material examined. Guizhou: 4 exs., , Guiyang, 15.viii.1982, lgt. Zhi-he Huang; 1 ex., Guiyang, 6.x.1940, lgt. Zhe-long Pu; 1 ex., Guiyang, Pingba Horse farm, 13.viii.1982, lgt. Zhi-he Huang; 8 exs., Guiyang, Huaxi, 12.viii.1982, lgt. Zhi-he Huang (1 ex. CV). Sichuan: 3 exs., Emei Mt., 31.viii.1982, lgt. Zhi-he Huang (1 ex. CV). Zhejiang: 1 ex., Tianmushan Mt., 27-28.vii.2007, lgt. Feng-long Jia.

Additional material examined by Vondel: Sichuan: $1 \delta^{\uparrow}$, Wenjian Distr., Guanxian Co., 56 km NW Changdu, Qingcheng Shan, 975 m, 3053,8N, 103³2,8E, 13.vii.1999.lgt. A. Putz (CF). Yunnan: 15 exs., E. Weishan Mt., 1800-2500 m, $25^{\circ} 10^{\prime} \mathrm{N}, 100^{\circ} 21^{\prime} \mathrm{E}, 22-25 . v i .1992$, lgt. Vitkubáň (CV, NHMB); 36 exs. Shizong, 9-15.ix.2000, lgt. J. Bergsten (CN, CV). 
Distribution. Eastern Palaearctic species, known from several provinces in the east and south of China: Beijing, Chongqing, Guizhou, Hunan, Jiangsu, Shanghai, Sichuan, Yunnan, Zhejiang. New records for Sichuan and Zhejiang.

\section{Haliplus (Haliplus) latreillei sp. $\mathrm{n}$.}

urn:lsid:zoobank.org:act:1C3B87DA-E87E-40CA-B74D-2E537338AFEB

http://species-id.net/wiki/Haliplus_(Haliplus)_latreillei

Figs 1-6

Material examined. See type material in Systematics chapter.

Distribution. Endemic to China: Guizhou.

\section{Haliplus (Haliplus) regimbarti Zaitzev, 1908}

http://species-id.net/wiki/Haliplus_(Haliplus)_regimbarti

Material examined. Fujian: 1 ex., Nanjing County, Hexi town (in pool), 13.vii.2010, lgt. Feng-long Jia; 2 exs., Fuzhou, 14.vii.1958, lgt. Zhe-long Pu; 1 ex., same locality, 4.ix.1941. Guangdong: 15exs, , Guangzhou, Luogang, 20.iv.1958 (3 exs. CV); 11 exs., Danxiashan Mt., 27.v.2007, lgt. Feng-long Jia. Guangxi: 1 ex., Wuming, 17.vi.1977, lgt. Zhihe Huang; 2 exs., Yangshuo, 1985, lgt. Shou-jian Chen. Henan: 6 exs., , Xinyang, Jigongshan Mt., viii.1936; Hubei: 1 ex., , Wuchang, 17.v.1961, lgt. Zhe-long Pu. Hunan: 4 exs., Nanyue Mt., 4.ix.1941, lgt. Zhe-long Pu; 1 ex., Liyuan, 6.iii.1941, lgt. Zhe-long Pu; 2 exs., Shaanxi, Chang'an, Weiqu, 21.viii.1984, Zhi-he Huang. Jiangxi: 1 ex., , Jinggangshan, Ciping, 18.ix.2010, lgt. Shuang Zhao; 1 ex., Jiangxi, Jinggangshan, Jingzhushan Mt., 4.x.2010, lgt. Feng-long Jia; 24 exs., same locality, 25.iv.2011, lgt. Fenglong Jia \& Shuang Zhao; 11 exs. Lushan, 10.viii.1963, lgt. Zhe-long Pu; 2 exs., Lushan, 10.viii.1963, lgt. Zhe-long Pu. Yunnan: 3 exs., , Pohui, 9.x.1940, lgt. Zhe-long Pu.

Additional material examined by Vondel: Guizhou: 1 ex., Fodingshan, ganshi, 25 km S Shiquian, 1300 m, 5-9.vi.1997, lgt. Bolm; 4 exs. Leigongshan, Xijiang, 12001900 m, 29.v-2.vi.1997, lgt. Bolm (CV, NHMB). Jiangxi: 1q, "Sharp; China, Kia Kiang; Dr. Régimbart vidit 1898; Shaanxi: 1 ex., without precise locality data; 1 , Süd Schensi [no further locality data] (NMPC).

Distribution. Endemic and widespread in the south-eastern part of China: Anhui, Fujian, Guangdong, Guangxi, Guizhou, Henan, Hubei, Hunan, Jiangsu, Jiangxi, Shandong, Shaanxi, Taiwan, Yunnan, Zhejiang. New records for Guangxi, Hubei, Shaanxi and Yunnan. 


\section{Haliplus (Haliplus) ruficollis (De Geer, 1774)}

http://species-id.net/wiki/Haliplus_(Haliplus)_ruficollis

Figs $11-14$

Material examined. Xinjiang: $2 \widehat{\partial} \widehat{\partial}$, Yining, Yili river valley, 28.vii.2005, lgt. Ling

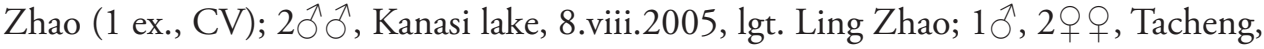
Hardun river bank, 4.viii.2005, lgt. Ling Zhao.

Distribution. A widespread western Palaearctic species reaching China in the utmost north-western part: Xinjiang. New to China.

\section{Haliplus (Haliplus) sibiricus Motschulsky, 1860}

http://species-id.net/wiki/Haliplus_(Haliplus)_sibiricus

Figs $15-18$

Material examined. Xinjiang: 32 exs., Yili Agricultral School, 3.viii.1964, lgt. Zhihe Huang (3 exs., CV); 9 exs., suburb of Wulumuqi, 16.vii.1984, lgt. Zhihe Huang; 6 exs., Wulumuqi, Liudaowan, 3.vii.1984, lgt. Zhihe Huang; 19 exs., Hongxing Farm, 6.viii.2005; 19 exs., Hot Spring, Swamp, 24.vii.2005, lgt. Ling Zhao; 3 exs., Gongnaisigou, 30.vii.2005; 7 exs., Altai, Xiaodonggou, 10.viii.2005, lgt. Ling Zhao; 5 exs. Tacheng, Hardun river bank, 4.viii.2005, lgt. Ling Zhao; 5 exs., Huocheng, river weir and river bank, 4.viii.2005, lgt. Ling Zhao; 4 exs., Kanasi lake, 8.viii.2005, lgt. Ling Zhao (2 exs., CV); 2 exs., Nalati steppe, 30.vii.2005, lgt. Ling Zhao.

Additional material examined by Vondel: Qinghhai: 6 exs. prov. Huangzhong env. Taer [lamasery], $36^{\circ} 28.8-29.5^{\prime} \mathrm{N}, 101^{\circ} 34.0-34.1^{\prime} \mathrm{E}, 2665-2780 \mathrm{~m}, 17 . v i i .2005$, lgt. J. Hájek, D. Král \& J. Růžička leg. (CV, NMPC)

Distribution. A widely spread western and north-eastern Palaearctic species, reaching the west of China: Qinghai, Xinjiang. New to China.

\section{Haliplus (Haliplus) simplex Clark, 1863}

http://species-id.net/wiki/Haliplus_(Haliplus)_simplex

Material examined. Heilongjiang: 1 ex., Liangshui, last third of July, 1977; 3 exs., Harbin, swampland, 29.vii.1962, lgt. Zhe-long Pu; 22 exs., same locality, 23.vii.1962; 1 ex., Harbin, 26.viii.1962, lgt. Zhe-long Pu; 3 exs., Wudalianchi, 6-10.viii.2008, lgt. Feng-long Jia. Inner Mongolia: 1 ex., Hailar, 23-26.vii.2003. Jilin: 2 exs., Gongzhuling, 5.viii.1962, lgt. Zhe-long Pu. Shaanxi: 16 exs., Chang'an, Weiqu, 21.viii.1984, lgt. Zhi-he Huang.

Additional material examined by Vondel: Anhui: 1 ex., [no further locality data], lgt. P. Brinck $(\mathrm{CN})$. Inner Mongolia: 3 exs., North Manchuria, Djalantun St. [Buth Qi], 6.v.1939, lgt. P. Brinck (CN). 


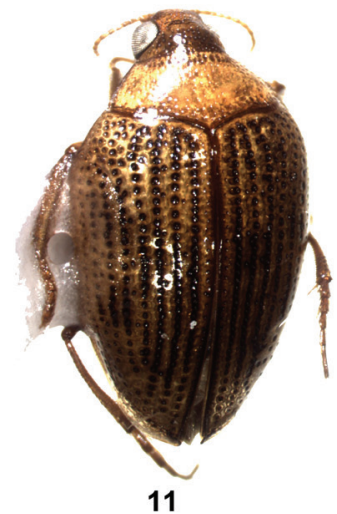

11

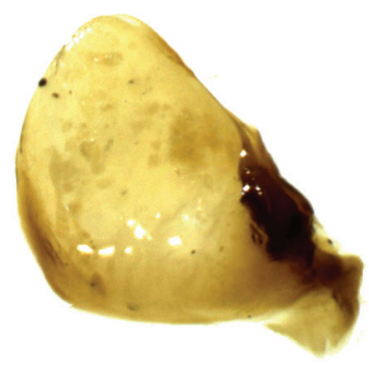

12

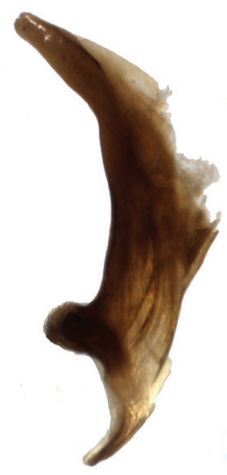

13

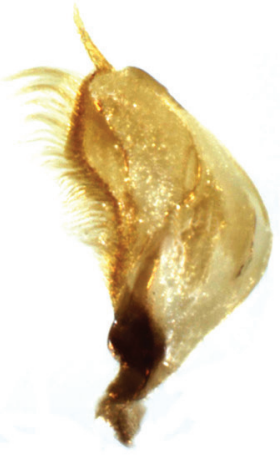

14

Figuress II-I4. Haliplus ruficollis II habitus I $\mathbf{2}$ left paramere $\mathbf{3}$ penis $\mathbf{4}$ right paramere.

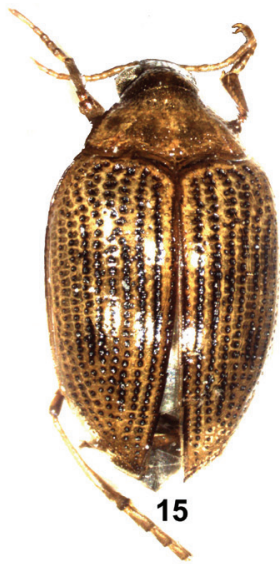

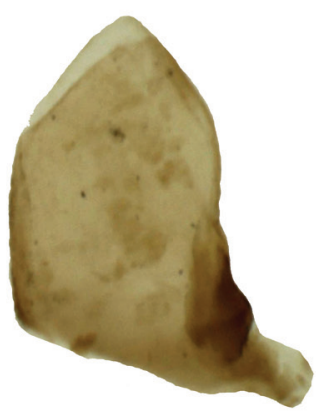

16

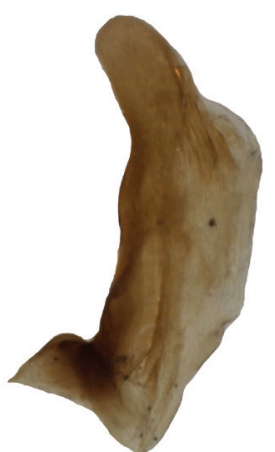

17

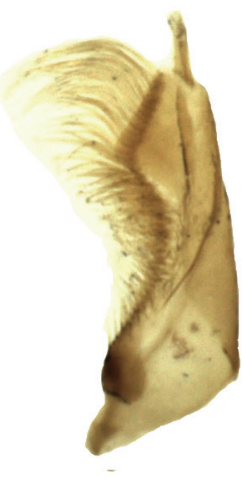

18

Figuress 15-18. Haliplus sibiricus $\mathbf{1 5}$ habitus $\mathbf{1 6}$ left paramere $\mathbf{1 7}$ penis $\mathbf{I 8}$ right paramere.

Distribution. Eastern Palaearctic species, known from several provinces in the eastern part of China: Anhui, Beijing, Guangdong, Heilongjiang, Inner Mongolia, Jiangsu, Jilin, Liaoning, Shaanxi, Shandong, Zhejiang. New records for Anhui, Inner Mongolia and Jilin.

12. Haliplus (Haliplus) steppensis Guignot, 1954

http://species-id.net/wiki/Haliplus_(Haliplus)_steppensis

Material examined. Heilongjiang: 1 ex, Liangshui, last third of July 1977. Inner Mongolia: 7 exs., Jining suburb, autumn, 1975 (2 exs., CV); 1 ex., Linhe, autumn, 1975; 3 exs., Hailar, 23-26.vii.2003, lgt. Feng-long Jia. 
Additional material examined by Vondel: Gansu: 15 exs., Dagcanglhamo [=Langmusi] env., 34 $04.7^{\prime} \mathrm{N}, 102^{\circ} 38.1^{\prime} \mathrm{E}, 3465 \mathrm{~m}$ alt, 23-25.vi.2005, leg. J. Hájek, D. Král \& J. Růžička (CV, NMPC). Qinghai: 4 exs., Haibu env. 3190-3270 m. 3648.449.8'N, 10045.4-49.7'E, 13-15.vii.2005, leg. J. Hájek, D. Král \& J. Růžička; 8exs., 7 km NE Ulan, 3020 m alt. 3657.6'N, 98³0.6'E, 7.vii.2005, leg. J. Hájek, D. Král \& J. Růžička (CV, NMPC).

Distribution. North-eastern Palaearctic species, known from the north of China: Gansu, Heilongjiang, Inner Mongolia, Qinghai. New records for Gansu, Inner Mongolia and Qinghai.

\section{Haliplus (Liaphlus) abbreviatus Wehncke, 1880}

http://species-id.net/wiki/Haliplus_(Liaphlus)_abbreviatus

Material examined. Xinjiang $1 \widehat{\jmath}$, Kuerle, Shayidong, 22.vii.1984, lgt. Zhihe Huang ; 1ठ̄, Kashi, 1.VIII.2006, lgt. Ling Zhao ; 1 q , Hetian, 16.vii.2006, lgt. Ling

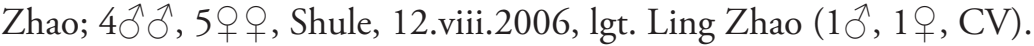

Note. The specimen from Kuerle was identified as Haliplus dalmatinus Müller, 1900 and reported as new to China by Jia (2003). The reexamination of this specimen shows that its previous identification was incorrect. Haliplus dalmatinus is removed from the Chinese list

Distribution. Southern Palaearctic reaching the western part of China: Xinjiang.

14. Haliplus (Liaphlus) basinotatus Zimmermann, 1924

http://species-id.net/wiki/Haliplus_(Liaphlus)_basinotatus

Material examined. Heilongjiang: 3 exs., Mishan, 26.viii.1964, lgt. De-ai Deng \& Shou-fa Hou; 1 ex., Harbin, 28.vii.1962, lgt. Zhe-long Pu.

Additional material examined by Vondel: 1 ex. Djalantun St., 6.v.1939. lgt. P. Brinck [Butha Qi, Inner Mongolia] (CN)

Distribution. North-eastern Palaearctic species, reaching China in the north-east: Heilongjiang, Inner Mongolia, Jilin, Liaoning.

15. Haliplus (Liaphlus) chinensis Falkenström, 1932

http://species-id.net/wiki/Haliplus_(Liaphlus)_chinensis

Material examined. Guizhou: 1 ex., Pingba Horse Farm, 13.vii.1982, lgt. Zhi-he Huang. Sichuan: 4 exs., , Dakang, 14.viii.2001, lgt. Ling Zhao.

Additional material examined by Vondel: Xinjiang: 1 q , Chingkiang, xi.1877 (NMPC). Yunnan: 5 exs., Shizong, 11-15.ix.2000, lgt. J. Bergsten (CN, CV) 
Note. The older records of $H$. ovalis Sharp, 1884 from China likely concern $H$. chinensis (for details see Vondel 1991, 2005)

Distribution. Endemic to China, known from several provinces from west to east: Beijing, Fujian, Guizhou, Inner Mongolia, Jiangsu, Shandong, Shanghai, Sichuan, Shanxi, Xinjiang, Yunnan, Zhejiang.

\section{Haliplus (Liaphlus) davidi Vondel, 1991}

http://species-id.net/wiki/Haliplus_(Liaphlus)_davidi

Material examined. Tianjin: 1 ex., Nanda, iv.1956.

Distribution. Eastern Palaearctic and Oriental species, known from the north and south of China: Beijing, Heilongjiang, Tianjin, Yunnan. New record for Tianjin.

\section{Haliplus (Liaphlus) diruptus J. Balfour-Browne, 1947}

http://species-id.net/wiki/Haliplus_(Liaphlus)_diruptus

Material examined. Guizhou: 1 ex., Guiyang, 9.x.1940, lgt. Zhe-long Pu. Heilongjiang: 1 ex., Wudalianchi, 6-10.viii.2008, lgt. Feng-long Jia. Hunan: 3 exs., Yizhang, 1.iv.1942, lgt. Zhe-long Pu; 2 exs., same locality, 10.ii.1941.Shaanxi: 1 ex., Chang'an, Wutaishan Mt., 23.viii.1984.

Additional material examined by Vondel: Anhui: 1 ex. Anhui [without further locality data], lgt. P. Brinck (CN). Guizhou: 1 ex., Fodingshan, Ganshi, 25 km S. Shiquian, $1300 \mathrm{~m}, 5-9 . v i .1997$, lgt. Bolm (NHMB). Yunnan: 10ં, $25 \mathrm{~km}$ E Zhongdian, 3300-4000 m, 12-14.vii.1995, lgt. Bolm (NHMB); 9 exs. Shizong, 10-13. ix.2000, lgt. J. Bergsten (CN, CV).

Distribution. Eastern Palaearctic and Oriental species, widely spread in the eastern part of China: Anhui, Beijing, Fujian, Guizhou, Hainan, Heilongjiang, Hong Kong, Hubei, Hunan, Jiangsu, Liaoning, Shaanxi, Shandong, Shanghai, Taiwan, Tianjin, Yunnan. New records for Anhui and Shaanxi.

18. Haliplus (Liaphlus) excoffieri Vondel, 1991

http://species-id.net/wiki/Haliplus_(Liaphlus)_excoffieri

Material examined. Guizhou: 1 ex., , Pingba Horse Farm, 13.vii.1982, lgt. Zhi-he Huang. Yunnan: 1 ex., Pohui, 9.x.1940, lgt. Zhe-long Pu.

Additional material examined by Vondel: Yunnan: 5 exs. Shizong, 12-13.ix.2000, lgt. J. Bergsten (CN, CV).

Distribution. Endemic species in the south of China: Guizhou, Yunnan. New record for Guizhou. 
19. Haliplus (Liaphlus) eximius Clark, 1863

http://species-id.net/wiki/Haliplus_(Liaphlus)_eximius

Material examined. Guangdong: 2 exs., Xingning, Luofu, Tieshan, 1.vii.2004, lgt. Feng-long Jia. Guizhou: 3 exs., Guiyang, Huaxi, 12.viii.1982, lgt. Zhi-he Huang. Xinjiang: 1 ex., Shule, 12.viii.2006, lgt. Ling Zhao. Yunnan: 1 ex., Lufeng village, 26.iii.1940, lgt. Zhe-long Pu.

Additional material examined by Vondel: Xinjiang: $1 \curvearrowright$, Chingkiang, xi.1877 [no further data](NMPC).

Distribution. Eastern Palaearctic and Oriental species, known from the west, east and south-east of China: Beijing, Fujian, Guangdong, Guizhou, Hunan, Jiangsu, Liaoning, , Shanghai, Sichuan, Xinjiang, Yunnan, Zhejiang. New record for Yunnan.

\section{Haliplus (Liaphlus) holmeni Vondel, 1991}

http://species-id.net/wiki/Haliplus_(Liaphlus)_holmeni

Distribution. Endemic to China: Yunnan.

\section{Haliplus (Liaphlus) kotoshonis Kano \& Kamiya, 1931}

http://species-id.net/wiki/Haliplus_(Liaphlus)_kotoshonis

Distribution. Eastern Palaearctic/Oriental species, known from the Ryukyu Islands (Japan) and Taiwan.

\section{Haliplus (Liaphlus) pulchellus Clark, 1863}

http://species-id.net/wiki/Haliplus_(Liaphlus)_pulchellus

Material examined. Guangxi: 2 exs., Nanning, 22.vi.1958, lgt. Zhe-long Pu.

Distribution. Oriental species, reaching China in the south: Fujian, Guangxi. New record for Guangxi.

23. Haliplus (Liaphlus) sharpi Wehncke, 1880

http://species-id.net/wiki/Haliplus_(Liaphlus)_sharpi

Material examined. (examined by Vondel): Anhui: 3 exs., Anhui, lgt. P. Brinck [no futher data] (CN). Guizhou: 10̂, Fodingshan, Ganshi, $25 \mathrm{~km}$ A Shiquian, $1300 \mathrm{~m}$, 5-9.vi.1997, lgt. Bolm (NHMB). Yunnan: 6 exs., Shizong, 10-13.ix.2000, lgt. J. Bergsten (CN, CV) 
Distribution. Eastern Palaearctic species, known from several provinces in the east and south of China: Anhui, Chongqing, Fujian, Guizhou, Liaoning, Shanghai, Taiwan, Yunnan.

\section{Peltodytes (Peltodytes) caesus (Duftschmid, 1805)}

http://species-id.net/wiki/Peltodytes_(Peltodytes)_caesus

Material examined. Xinjiang: 1 ex., Yili Agriculture School, 3.viii.1984, lgt. Zhi-he Huang; 1 ex., Yining, Yili river valley, 28.vii.2005; 1ex., Emin, Hongxing Farmland, 6.viii.2005, lgt. Ling Zhao (1 ex., CV); 1 ex., Tacheng, Hardun river bank, 4.viii.2005, lgt. Ling Zhao.

Distribution. Widely spread Palaearctic species, reaching to China in the utmost north-west: Xinjiang.

\section{Peltodytes (Peltodytes) coomani Peschet, 1923}

http://species-id.net/wiki/Peltodytes_(Peltodytes)_coomani

Material examined. Guangdong: 1 ex., Guangzhou, Kangle (Sun Yat-sen University campus), iv.1959, lgt. Ping Lin; 1 ex., same locality, 24.vii.1964, lgt. Jiu-ru Zhang. Guangxi: 1 ex., Nanning, iv.1959, lgt. Zhe-long Pu; 2 ex., Nanning, vi.1958, lgt. Zhelong Pu (2 ex., CV). Hainan: 1 ex., Dongfang, Huangliu, 25.xii.1963, lgt. Tong-xu Peng; 2 exs., Tongshi, 27.xii.1963, lgt. Tong-xu Peng; 1 ex., Wanning County, Xinglong, 3.i.1964, lgt. Tong-xu Peng; 2 exs., Jianfengling Mt., 19.xii.1963, lgt. Tong-xu Peng.

Distribution. Oriental species, reaching to China in the south-east: Guangdong, Guangxi, Hainan. New record for Guangxi.

\section{Peltodytes (Peltodytes) dauricus Zimmermann, 1924}

http://species-id.net/wiki/Peltodytes_(Peltodytes)_dauricus

Material examined. Heilongjiang: $1 \hat{\jmath}$, Mishan, 1.x.1959.

Additional material examined by Vondel: Inner Mongolia: 5 exs., Djalantun [Buth Qi], 6.v.1939, lgt. P. Brinck (CN). Heilongjiang: 3 exs., Harbin, Manchoukuo, 4.x.1937, lgt. M.A. Weymarn; 1 ex., Harbin, Manchuria, 1938, lgt. M.I. Nikitin (SEMC). Liaoning: 1 ex., Korea, Daireu [=Dalian Shi, Liaoning], 1-15.ix.1937, lgt. Weymarn $(\mathrm{CN})$.

Distribution. North-eastern Palaearctic species, reaching to China in the northeast: Heilongjiang, Inner Mongolia, Liaoning. 


\title{
27. Peltodytes (Peltodytes) intermedius (Sharp, 1873)
}

http://species-id.net/wiki/Peltodytes_(Peltodytes)_intermedius

Material examined. Sichuan: 1 d, Emeishan Mt., 6.viii.1982, lgt. Zhi-he Huang. Guangdong: $1 \hat{\jmath}$, Guangzhou, Luogang cave, 12.x.1932, lgt. Y.W. Diou.

Distribution. Eastern Palaearctic species, known from the south-east of China: Beijing, Fujian, Guangdong, Shanghai, Sichuan, Taiwan, Zhejiang. New records for Sichuan and Guangdong.

\section{Peltodytes (Peltodytes) pekinensis Vondel, 1992}

\author{
http://species-id.net/wiki/Peltodytes_(Peltodytes)_pekinensis
}

Material examined. Guangdong: 1 ex., the second worker's culture palace, x.1955. Shaanxi: 4 exs. Xi'an Chanba river, 11.v.2011, lgt. Fenglong Jia; 6 exs. same locality, lgt. Hájek (NMPC). Tianjin: 1 ex., Nanda, iii.1956.

Distribution. Eastern Palaearctic species, known from the most-eastern part of Russia and several provinces in the east of China: Beijing, Fujian, Guangdong, Hebei, Liaoning, Shaanxi, Shandong, Tianjin. New records for Guangdong, Shaanxi and Tianjin.

\section{Peltodytes (Peltodytes) sinensis (Hope, 1845)}

http://species-id.net/wiki/Peltodytes_(Peltodytes)_sinensis

Material examined. Chongqing: 1 ex., Nanchuan, Tianxing, 27.vii.2003, lgt. Jianhua Huang. Fujian: 1 ex., , Fuzhou, 14.vii.1956, lgt. Zhe-long Pu; 1 ex., Fuzhou, Gushan, 14.vii.1956, lgt. Li-zhong Hua; 7 exs., Fuzhou, Xihu, 3.xi.1963, lgt. Shanxiang Lin. Guangdong: 1 ex., Lianxian, vi.1945, lgt. Zhe-long Pu; 1 ex., Shaoguan, Yingde, 4.viii.1962, lgt. Ping Lin; 1 ex., Sihui, Dasha, 6.iii.1998, lgt. Feng-long Jia; 1 ex., Heshan, 18.3.1994, lgt. Feng-long Jia; 1 ex., Lianshan County, Shangshuai, 4.v.2000, lgt. Feng-long Jia; 1 ex., Honan Island, 19.iii.1938, lgt. Chiu-an Wang; 1 ex., Zhaoqing, 12.x.1974, Meiying Wang; 1 ex., Qujiang, date for collection could not be read, lgt. Zhe-long Pu; 1 ex., Guangzhou, Ershatou, 11.iv.1958; 2 exs., Fengkai, Heishiding Mt., 29.v.1984, lgt. Zhi-he Huang; 3 exs., Lianxian, Dadongshan Mt., 16.ix.1993, lgt. Feng-long Jia; 11 exs., Xingning, Luofu, Tieshan, 1.vii.2004, lgt. Feng-long Jia; 1 ex., Danxiashan Mt., 23.v.2008, lgt. Feng-long Jia; 1 ex., Puning, 1958, lgt. Zhe-long Pu; 15 exs., Huaxian (Huadu) Dabuling, 26.viii.1983, lgt. Zhihe Huang; 2 exs., Guangzhou, Baiyunshan, 18.iv. 1958, lgt. Zhe-long Pu. Guangxi: 6 exs., , Yangshuo, 1958, lgt. Shoujian Chen et. al.; 1 ex., Longlin, 22.v.1977, lgt. Zhi-he Huang; 4 exs., Wuming, 7.vi.1977, lgt. Zhi-he Huang; 1 ex.m Shangsi, 24.vii.1977, lgt. Zhi-he Huang; 1 ex., Huaping, 25.vi.1974, lgt. De-xiang Gu; 1 ex., Jingxi, Ban- 
gliang, 31.vii.2010, lgt. Jian-hua Huang. Guizhou: 1 ex., Guiyang, 6.x.1940, lgt. Zhe-long Pu; 3exs., Guiyang, 12.viii.1982, lgt. Zhi-he Huang; 3 exs., Pingba Horse Farm, 13.viii.1982, lgt. Zhi-he Huang (1 ex., CV); 1 ex., Guiyang, 15.viii.1982, lgt. Zhi-he Huang; 1 ex., Fanjingshan, half of mount, 29.vii.2001, lgt. Hong Pang; 1 ex., Rongxian County, Pingyang, Xiaodanjiang river, 685m, 15.ix.2005, lgt. Shuang Zhao; 2 exs., , Guiyang, 6.x.1940, lgt. Zhe-long Pu. Hubei: 18 exs., Zigui, Jiutouling, 150m, 20.vii.1993, lgt. Xing-ke Yang \& Wen-zhu Li; 2 exs., Badong, Sanxia Forest workshop, 160m, 30.vii.1993, lgt. Xing-ke Yang \& Wen-zhu Li; 1 ex., Wuchang, vi.1958, lgt. Jieyue Hu. Hunan: 2 exs., Nanyue Mt., 4.ix.1941, lgt. Zhe-long Pu; 16 exs., Daotong, 19.viii.1982, lgt. Zhi-he Huang; 1ex., Huaihua, 17.viii.1982, lgt. Zhihe Huang; 1 ex., Yizhang, 8.x.1941, lgt. Zhe-long Pu; 1 ex., same locality, 10.ii.1941; 10 exs., Huaihua, Yushuwan, 17.vi.1965, lgt. Zhen-yao Chen; 4 exs., Qingjiang, Anjiang, 20.vi.1956, lgt. Zhen-yao Chen. Jiangxi: 2 exs., Jingu County, 22.viii.1974; 6 exs., Nanchang, viii.1957, lgt. Xi-wen Chen; 2 exs., samelocality, 26.viii.1963, lgt. Zhe-long Pu; 2 exs., Shangrao, Sanqingshan Mt., 15-20.iv.2007, lgt. Feng-long Jia; 2 exs., Longnan, Jiulianshan Mt., 12-13.vii.2008, lgt. Feng-long Jia; 3 exs., Jiujiang, Changdu, Linshan, 15-20.viii.2010, lgt. Yan Mei; 1 ex., Lushan, Poyang Lake, 10.viii.1963, lgt. Zhe-long Pu; 1ex., Nanchang, no other data; 9 exs., Yongxin County, 19.viii.1974; 3 exs., Fuzhou, 16-18.viii.1974; 1 ex., , Ji'an, 13.viii.1974; 1 ex., Jinggangshan, 16-18.viii.1974; 2 exs., Jinggangshan, Shuangxikou, 13.x.2010, lgt. Feng-long Jia; 4 exs. Jinggangshan, Baiyinhu, 800 m, 27.iv.2011. Shaanxi: 17 exs., Chang'an Weiqu, 21.viii.1984, lgt. Zhi-he Huang; 1ex., Xi'an Wujiafen, 17.viii.1984, lgt. Zhi-he Huang; 2 exs., South of Chang'an, Wutai, 23.viii.1984, lgt. Zhi-he Huang; 1 ex., Zhenba, 20.vii.1975, lgt. Shuzhi Ren. Sichuan: 3 exs., Qingchengshan Mt., 8.viii.1982, lgt. Zhi-he Huang; 7 exs., Wanxian, Longju, 450m, 14.vii.1993, lgt. Wen-zhu Li; 4 exs., Wanxian, Wang'erbao, 1200m, 9.vii.1993, lgt. Wen-zhu Li; 1 ex., Fengdu, 200m, 1.vi.1994, You-wei Zhang; 8 exs., Emeishan Mt., 31.vii.1983, lgt. Zhi-he Huang ( 3 exs., CV); 2 exs., Dakang, 14.viii.2001, lgt. Ling Zhao; 1 ex. Xi'an, Chanba river, 11.iv.2011, lgt. Fenglong Jia; 3 exs. same data, but J. Hájek lgt. (NMPC). Tianjin: 1 ex., Nanda, iv.1956. Yunnan: 4exs., Pohui, 9.x.1940, lgt. Zhelong Pu; 1 ex., Lijiang, v.2007, lgt. Run-lin Xu.

Additional material examined by Vondel: Anhui: 3 exs., Anhui [no further data], lgt. P. Brinck (CN). Fujian: Kuatun, 1946 (NHMB). Guangxi: 1 ex., 20 km N Lingchuan, $500 \mathrm{~m}, 21-24 . v i .1997$, lgt. Bolm (NHMB). Jiangxi: 1 ex., $5 \mathrm{~km}$ N Daduan town, $114^{\circ} 35^{\prime} 53^{\prime \prime E}, 28^{\circ} 36^{\prime} 30^{\prime \prime N}$, ca 450 m, 29.iii.2003, lgt. Schönmann, Komarek \& Wang (NMW). Shanghai: 1 ex., Kiangsee Jangtzi, Shanghai Xanthus [no further data] (CA). Yunnan: 73 exs. Shizong, 9-15.ix.2000, lgt. J. Bergsten (CN, CV)

Distribution. Eastern Palaearctic and Oriental species, widely spread in the eastern and south-eastern part of China: Anhui, Beijing, Chongqing, Fujian, Guangdong, Guangxi, Guizhou, Hainan, Hebei, Henan, Hubei, Hunan, Jilin, Jiangsu, Jiangxi, Liaoning, Shaanxi, Shandong, Shanghai, Sichuan, Taiwan, Yunnan, Zhejiang. New records for Chongqing, Hubei and Shaanxi. 


\section{Acknowledgements}

We are grateful to Miss Shuang Zhao for her help with preparing photographs. This work was supported by "Biodiversity of Mount Jinggangshan in China" (2010330007102993).

\section{References}

Balfour-Browne J (1947) The aquatic Coleoptera of Manchuria (Weymarn collection). Annals and Magazine of Natural History (11) 13(1946): 433-460.

Jia FL (2003) Two New Record Species of Haliplidae (Coleoptera) from China. Entomotaxonomia 25(3): 178-180.

Holmen M (1987) The aquatic Adephaga (Coleoptera) of Fennoscandia and Denmark I. Gyrinidae, Haliplidae, Hygrobiidae and Noteridae. Fauna Entomologica Scandinavica 20: 1-169.

Makhan D (1999) Three new species of Haliplidae (Coleoptera) from China. Entomotaxonomia 21(4): 269-274.

Štastný J, Boukal M (2003) Haliplidae: III. Haliplus (Haliplidius) rejseki sp. n. From Sichuan (Coleoptera). In: Jäch MA, Ji L (Eds) Water Beetles of China, Vol. III. Wien: ZoologischBotanishe Gesellschaft in Österreich and Wiener Coleopterologenverein, 295-300.

Vondel BJ van (1990) Description of Haliplus harminae n. sp. from China (Coleoptera: Haliplidae). Entomologische Berichten, Amsterdam 50(2): 22-24.

Vondel BJ van (1991) Revision of the Palaearctic species of Haliplus subgenus Liaphlus Guignot (Coleoptera: Haliplidae). Tijdschrift voor Entomologie 134: 75-144.

Vondel BJ van (1992) Revision of the Palaearctic and Oriental species of Peltodytes Régimbart (Coleoptera: Haliplidae). Tijdschrift voor Entomologie 135: 275-297.

Vondel BJ van (1993) Revision of the Liaphlus species of the Oriental region, excluding China (Coleoptera: Haliplidae). Tijdschrift voor Entomologie 136: 289-316.

Vondel BJ van (1995) Haliplidae: Review of the Haliplidae of China (Coleoptera). In: Jäch MA, Ji L (Eds) Water Beetles of China, Vol. I. Wien: Zoologisch-Botanische Gesellschaft in Österreich and Wiener Coleopterologenverein, 111-154.

Vondel BJ van (1997) Haliplidae. In: Schwoerbel J, Zwick P (eds) Süßwasserfauna von Mitteleuropa 20(2): 1-95, 49 Figs

Vondel BJ van (1998) Haliplidae: Additional notes on the Haliplidae of China and neighouring countries (Coleoptera). In: Jäch MA, Ji L (Eds) Water Beetles of China, Vol. II. Wien: Zoologisch-Botanische Gesellschaft in Österreich and Wiener Coleopterologenverein, 131-136.

Vondel BJ van (2003a) Haliplidae: II. Additional notes on Chinese Haliplidae, with description of a new species (Coleoptera). In: Jäch MA, Ji L (Ed) Water Beetles of China, Vol. III. Wien: Zoologisch-Botanische Gesellschaft in Österreich and Wiener Coleopterologenverein, 289-294. 
Vondel BJ van (2003b) Haliplidae: I. Three new synonymies (Coleoptera). In: Jäch MA, Ji L (Eds) Water Beetles of China, Vol. III. Wien: Zoologisch-Botanische Gesellschaft in Österreich and Wiener Coleopterologenverein, 285-287.

Vondel BJ van (2005) Haliplidae. In: Nilsson A, Vondel BJ van: World Catalogue of Insects Vol. 7. Amphizoidae, Aspidytidae, Haliplidae, Noteridae and Paelobiidae (Coleoptera, Adephaga). Apollo Books, Stenstrup, 20-86.

Vondel BJ van (2007) World Catalogue of Haliplidae - corrections and additions, 1 (Coleoptera: Haliplidae). Koleopterologische Rundschau 77: 89-96.

Vondel BJ van (2010) Revision of the Haliplidae of the Afrotropical region including North Africa (Coleoptera). Tijdschrift voor Entomologie 153: 239-314 + Figs 1-326.

Vondel BJ van, Holmen M, Petrov PN (2006) Review of the Palaearctic and Oriental Species of the subgenus Haliplus s. str. (Coleoptera: Haliplidae: Haliplus) with description of three new species. Tijdschrift voor Entomologie, 149: 227-273.

Vondel PJ, Spangler PJ (2008) Revision of the Haliplidae of the Neotropical Region including Mexico (Coleoptera: Haliplidae). Koleopterologische Rundschau 78: 69-194.

Watts CHS, McRae J (2010) The identity of Haliplus (Coleoptera: Haliplidae) from the Pilbara region of Australia, including the description of four new species. Records of the Western Australian Museum 25: 387-398.

Wu CF (1937) Haliplidae. In: Wu CF (Ed) Catalogus Insectorum Sinensium III, Peiping: The Fan Memorial Institute of Biology, 193-196. 